\title{
Birth attendants' hand hygiene compliance in healthcare facilities in low and middle- income countries: a systematic review
}

\author{
Giorgia Gon ${ }^{1 *}$ (D) Mícheál de Barra², Lucia Dansero ${ }^{3}$, Stephen Nash ${ }^{1}$ and Oona M. R. Campbell ${ }^{1}$
}

\begin{abstract}
Background: With an increasing number of women delivering in healthcare facilities in Low and Middle Income Countries (LMICS), healthcare workers' hand hygiene compliance on labour wards is pivotal to preventing infections. Currently there are no estimates of how often birth attendants comply with hand hygiene, or of the factors influencing compliance in healthcare facilities in LMICs.

Methods: We conducted a systematic review to investigate the a) level of compliance, b) determinants of compliance and c) interventions to improve hand hygiene during labour and delivery among birth attendants in healthcare facilities of LMICs. We also aimed to assess the quality of the included studies and to report the intracluster correlation for studies conducted in multiple facilities.

Results: We obtained 797 results across four databases and reviewed 71 full texts. Of these, fifteen met our inclusion criteria. Overall, the quality of the included studies was particularly compromised by poorly described sampling methods and definitions. Hand hygiene compliance varied substantially across studies from 0 to 100\%; however, the heterogeneity in definitions of hand hygiene did not allow us to combine or compare these meaningfully. The five studies with larger sample sizes and clearer definitions estimated compliance before aseptic procedures opportunities, to be low (range: 1-38\%). Three studies described two multi-component interventions, both were shown to be feasible.

Conclusions: Hand hygiene compliance was low for studies with larger sample sizes and clear definitions. This poses a substantial challenge to infection prevention during birth in LMICs facilities. We also found that the quality of many studies was suboptimal. Future studies of hand hygiene compliance on the labour ward should be designed with better sampling frames, assess inter-observer agreement, use measures to improve the quality of data collection, and report their hand hygiene definitions clearly.
\end{abstract}

Keywords: Hand hygiene, Maternal and newborn health, Labour, Healthcare workers

\footnotetext{
* Correspondence: giorgia.gon@lshtm.ac.uk

'London School of Hygiene and Tropical Medicine, Keppel Street, London WC1E 7HT, UK

Full list of author information is available at the end of the article
} 


\section{Background}

Globally, infection contributes to at least $9 \%$ of maternal deaths [1] and $16 \%$ of neonatal deaths [2], the vast majority of this burden concentrates in low and middle income countries (LMICs). Hand hygiene during birth has been long recognised as a key infection prevention opportunity [3, 4]. With an increasing number of women delivering in healthcare facilities in LMICs [5], appropriate hand hygiene compliance of healthcare workers on the labour wards is pivotal to preventing infections.

Several systematic reviews have been published on the compliance, determinants and interventions to improve healthcare workers hand hygiene across the facility environment [6-10]; only two of these reviews include studies from low resource healthcare facilities, none of which provide estimates for the labour ward [7, 8]. Erasmus et al. report a median hand hygiene compliance of $40 \%$ for studies from high-income countries [6]; the other, more recent, reviews focus on evaluating existing interventions and do not report summary estimates of compliance, but there is value in collating estimates from observational studies too.

Currently there are no estimates of how often birth attendants comply with hand hygiene, or of the factors influencing their compliance in healthcare facilities in LMICs. Hand hygiene compliance in LMICs may differ in levels and determinants compared to those in high-income countries (HICs), where most published evidence is. For example, there are cultural and contextual elements around the process of labour and delivery that might influence hand hygiene compliance of healthcare workers such as unpredictable workloads, unreliable water supplies, or the concept of pollution and purity around delivery - important among healthcare workers in India and Bangladesh $[11,12]$. Finally, detailed estimates on compliance in LMICs and their determinants are useful to inform whether interventions are needed, and how to tailor them.

The aim of this paper is to systematically review the literature from LMICs to:

1. Estimate birth attendants' hand hygiene compliance during labour and delivery in healthcare facilities

2. Assess the quality of the studies reporting these estimates

3. Investigate what factors influence hand hygiene compliance

4. Estimate the effectiveness of interventions aimed at increasing hand hygiene compliance

5. Estimate intra-cluster correlation for hand hygiene compliance comparing variation within and between facilities

\section{Methods}

The search was conducted on the $1^{\text {st }}$ of September 2020, updating earlier searches on the 24th of April 2018 and on the 27th of January 2016 over EMBASE, MEDLINE, CINHAL, and the WHO regional databases (the website we used for the latter was not accessible during the last search in spite several attempts). We used a comprehensive set of search terms based on previous systematic reviews $[8,13,14]$ and consulted the London School of Hygiene and Tropical Medicine librarian. The search themes included hand hygiene and maternity ward terms with international spelling variations, and it was restricted to LMICs. Additional file 1 details the strategy. Peer reviewed articles were eligible for inclusion, while abstracts and conference proceeding were not. All texts were reviewed using Endnote X7. No protocol was registered for this review.

Duplicates were removed, and titles and abstracts screened for any mention of hand hygiene compliance in labour wards. Two reviewers independently applied the inclusion criteria to the selected full texts. Any discrepancy was resolved through discussion. Once full texts were selected, one author screened references to search for other relevant studies that might be eligible for inclusion. The inclusion criteria were:

- Studies with either of the following estimates for the specific group of healthcare workers attending labour and delivery or working on the labour ward:

- A measure of frequency for hand hygiene compliance (observed or other objective method; self-reports were not included)

- OR an effect size (odds ratio, rate ratio, risk ratio) of factors driving hand hygiene (observed or other objective method; self-reports were not included)

- LMICs based studies

- Peer-reviewed studies

- Intervention or observational studies

- Quantitative studies

- Studies in any language

Data extraction was done by one author and checked by another. The data extraction form included study type, intervention details, country, urban-rural location, type of healthcare facility, staff cadre, facility ward specification, availability of hand hygiene infrastructure (soap, water, handrub), sample size, sample selection, analysis methods, measurement tools, and the effect size of hand hygiene determinants. We extracted the estimates of hand hygiene compliance by healthcare workers before aseptic procedures (or compliance estimates which were likely to include before aseptic procedure opportunities) for a) types of patient-attendant interactions that could 
occur during labour and delivery, or b) healthcare workers working in the labour ward. We specifically focused on estimates reflecting hand hygiene opportunities before aseptic procedures these because these are the most pivotal to infection prevention. For each estimate we extracted the hand hygiene definition, the numerator, denominator, the percentage compliance estimates, the number of staff or women observed, the staff cadre, the number of facilities, and the intervention stage details underpinning the individual estimate. We calculated the percentage compliance for each included study where this was possible. We contacted the corresponding author (or if this was not published, the first or senior author whose email we found via their department or on researchgate) when it was not clear from the paper whether a) their observation included procedures around labour and vaginal delivery; or b) when the hand hygiene definition was unclear and the tool used was not available.

Key measures of bias and quality were included in the data extraction. For randomised controlled trials we intended to use the CONSORT guidelines to assess quality. For observational studies, we assessed quality using checklist we developed using eight items adapted from the STROBE guidelines' [15] methods section (as recommended by Sanderson and colleagues) [16], to the specific context of observing hand hygiene in healthcare settings. Items included assessing 1) sampling methods, 2) quality of data collection, 3) description of the data collectors background, 4) whether inter-observer agreement was estimated, 5) the definition of hand hygiene compliance, 6) details of the tool used for observation, 7) whether study aims were concealed from the study participants and 8) whether the statistical procedures were described. Items were scored positively or negatively, except for items 1,3 and 6 where we added an extra option of partially met when only one of two criteria was met, and item 7 which could also be scored as unclear.

Intra-cluster correlation (ICC) accounts for the relatedness of data by comparing the variance within clusters with the variance between clusters; it is useful for designing and analysing observational and intervention studies. To obtain the ICC for hand hygiene compliance of the included studies comparing the variation in compliance between and within facilities, we also contacted the authors of studies with multiple facilities (clusters) to ask for:

- Either, the following single measures:

- The standard deviation exhibiting how the cluster means vary from the population mean from cluster to cluster $\sigma_{b}$ (between-cluster variation)
- The standard deviation exhibiting how individual values vary from their cluster mean from individual to individual $\sigma_{w}$ (within-cluster variation). Individuals are birth attendants in our review.

- Or, the overall estimated ICC $(\rho)=\rho=\sigma_{b}^{2} /\left(\sigma_{b}^{2}+\right.$ $\left.\sigma_{w}{ }^{2}\right)$

We aimed to conduct pooled analysis of the estimates by hand hygiene compliance estimated using similar outcome definitions, measurement tools or investigating similar interventions, unless there are differences in setting or risk of bias; where studies did not use similar outcomes, measurement tools or investigate similar interventions, estimates were described.

We followed the PRISMA guidelines for systematic reviews to report our methods and findings (see Additional file 2) [17].

\section{Results}

After removing duplicates (100), we obtained 697 results across the four databases and reviewed 71 full texts of which 4 are from reference searching (Fig. 1). We ultimately included fifteen that met our inclusion criteria. The reasons for excluding the fifty-seven studies are in Fig. 1, with the most common being that the study did not report on the outcome of interest, i.e. hand hygiene of healthcare workers during labour or delivery, or in the labour ward. In two articles which were identified via reference searching, it was unclear whether labour and delivery were being studied, and the author of the paper did not reply to enquiry, so these papers were not included.

Of the fifteen included studies, seven were in SubSaharan Africa (Zanzibar-Tanzania, Zimbabwe, two in Ghana, and three in Nigeria), two were in Iran, the rest were located in in South-East Asia: three in India, one in Vietnam, one in the Thai-Myammar border, and one spanned several countries (Cambodia, Lao People's Democratic Republic, Mongolia, Papua New Guinea, Philippines, Solomon Island, and Vietnam) - see Table 1. The studies were published between 1993 and 2020, with only one study being published prior to 2008. Four studies were conducted in a single facility. Six of the nine studies did not report any information on hand hygiene infrastructure (Table 1); one study discussed how inconvenient the sink location was; one study selected the hospital based on it generally having supplies to provide good quality of maternal care; three studies reported on the general availability of supplies (two positively and one negatively), but it is unclear what elements of hand hygiene infrastructure were surveyed if any. Only four studies reported specifically on the availability of hand hygiene infrastructure. Two of these 


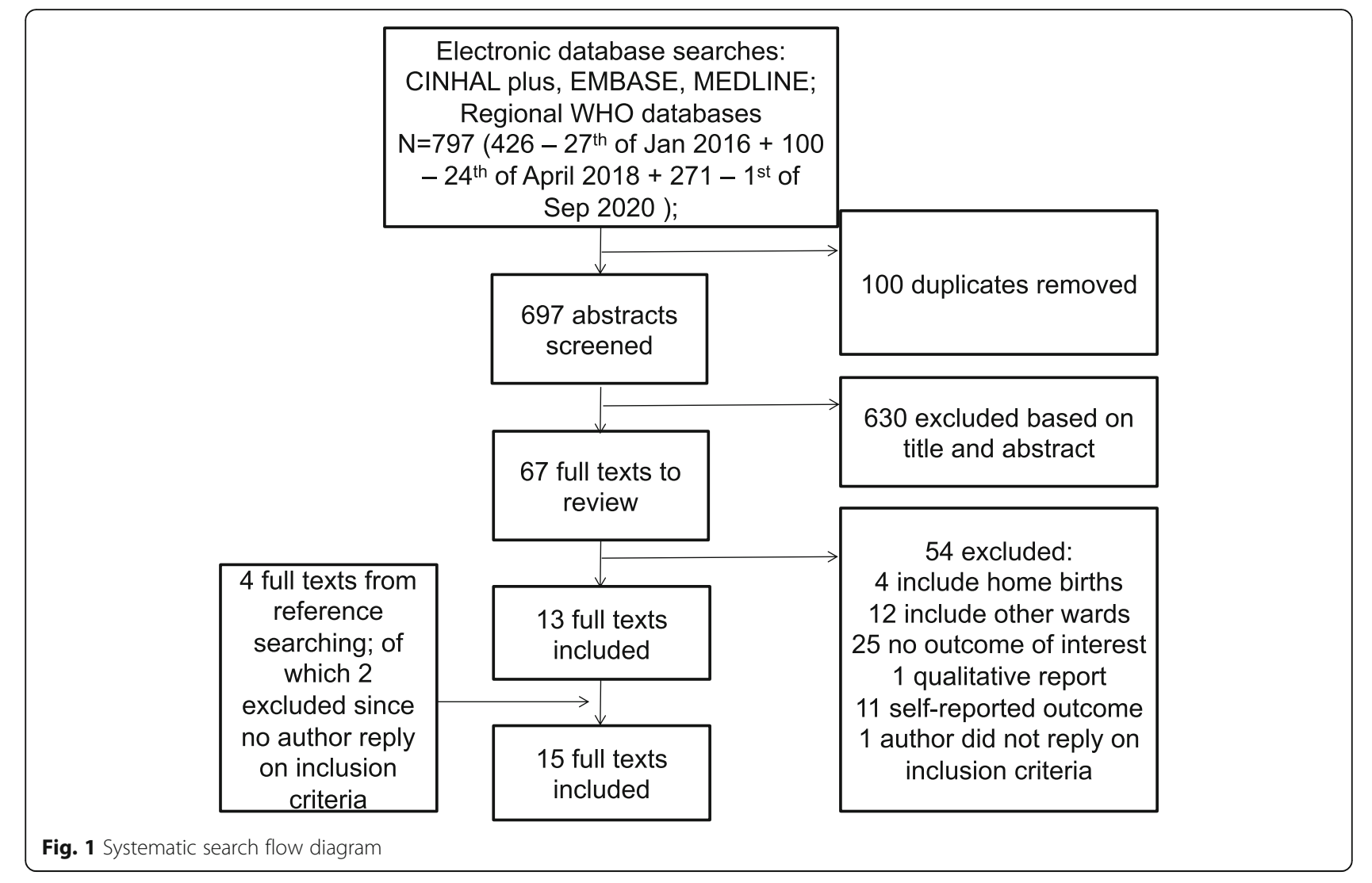

studies reported that needed supplies were present, except for handrub in the first study [32], and disposable towel in the second [19]; one reported that not all the facilities had needed supplies, but the percentage refers to a wider set of facilities compared to the one observed for hand hygiene [27]; and one reported the availability of $24-\mathrm{h}$ running water (52\% of facilities) and soap ( $65 \%$ of facilities) (Table 1) [24].

\section{Quality of primary studies}

All studies used observation as their primary method of data collection. The methods were described in most articles only partially. The lowest ranked quality indicators were 1) sampling, 2) methods to enhance data quality during data collection, 3) measurement of inter-observer agreement, and 4) the level of description of the hand hygiene compliance definition - see Fig. 2.

\section{Sampling}

We required two aspects of the sampling methods to be described: a) how the unit of observation (e.g. woman, procedure or healthcare worker) was sampled and b) how the timing of facility visits were scheduled. None described both aspects sufficiently; five articles did not describe them at all. As detailed in Table 1, it was often unclear how different women or healthcare workers were selected for observation.

\section{Quality during data collection}

Only four articles directly addressed the procedures adopted to ensure a better quality of data collection. Buxton et al. report that data collection did not start until results were consistent during the training period [19]. Spector et al. included on-site reviews of all observation forms within $72 \mathrm{~h}$ by the local study coordinator, and in-built data management checks confirming the data collected were logical [30]. Gon et al. provided tailored feedback to data collectors based on the results of the inter-observer exercise run in the first month [25]. Tyagi et al. incorporated quality checks in their tool as a results of the training [31].

\section{Inter-observer agreement}

Gon et al. is the only study that reports the results of interobserver agreement. This was calculated between pairs of data collectors in the first month of the study; the range of kappa statistics results was 0.73-0.93 for three pairs of data collectors [25]. Buxton et al. report that inter-rater reliability was monitored during the training period but do not report their results [19]. 


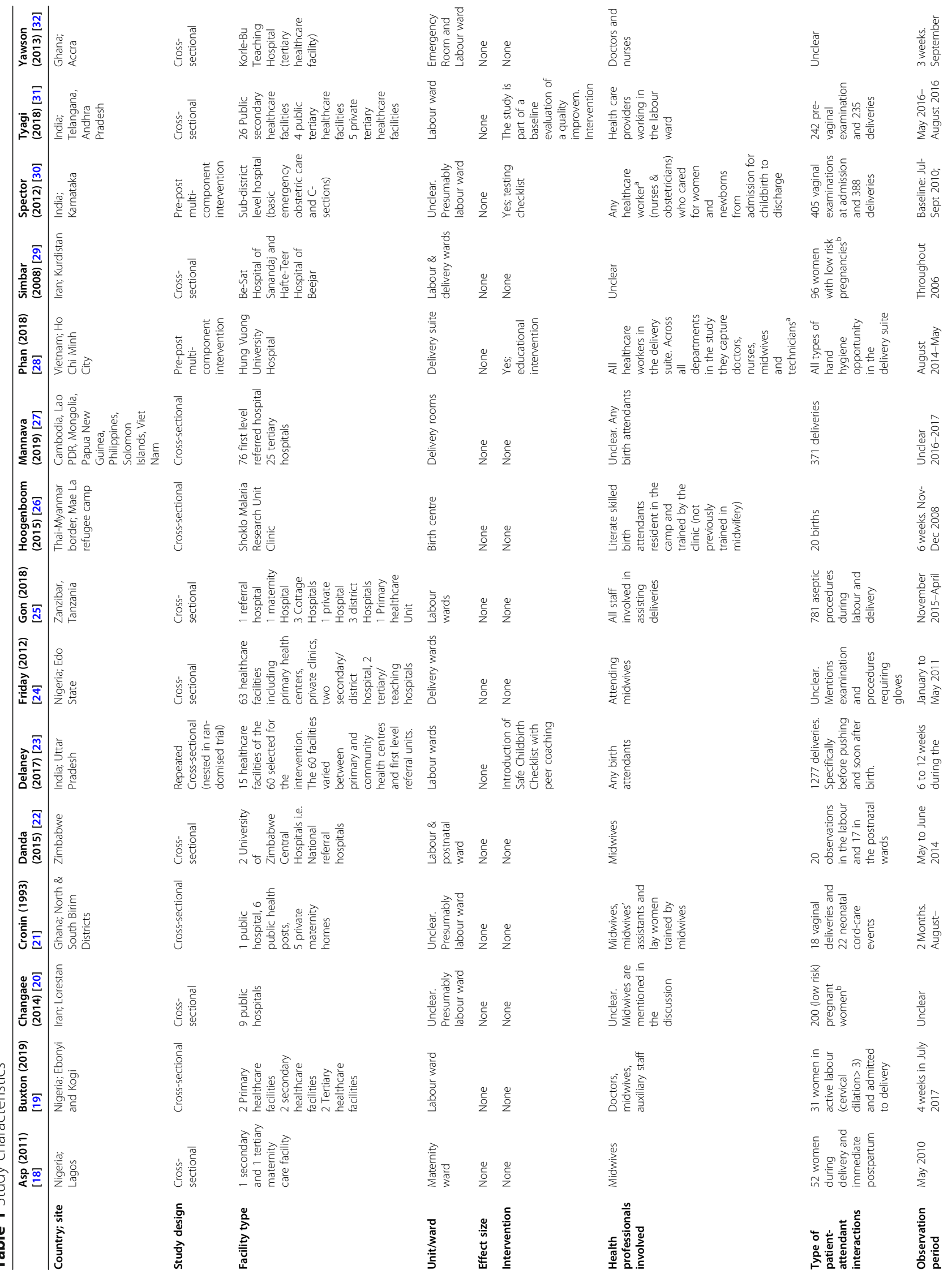




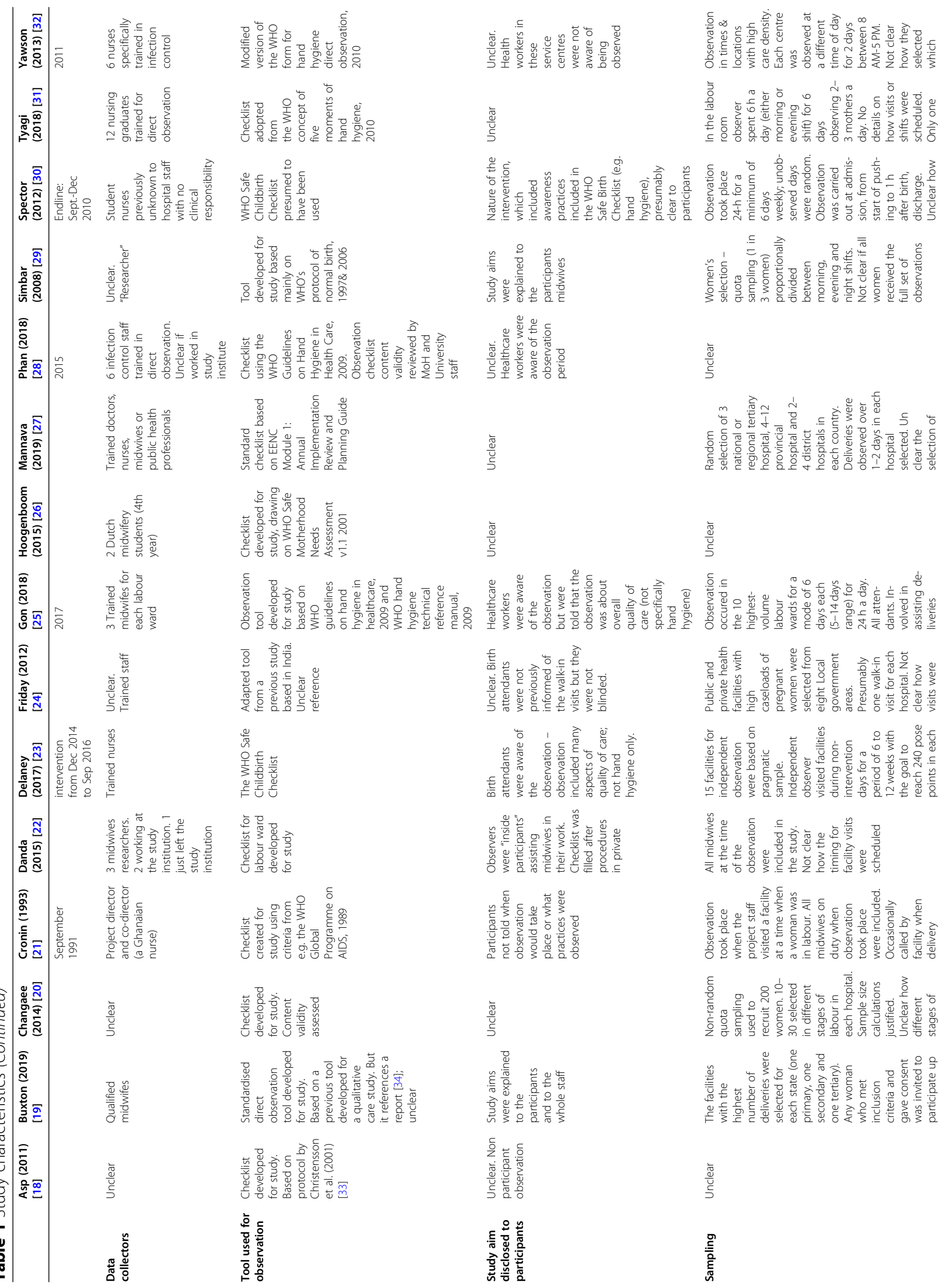




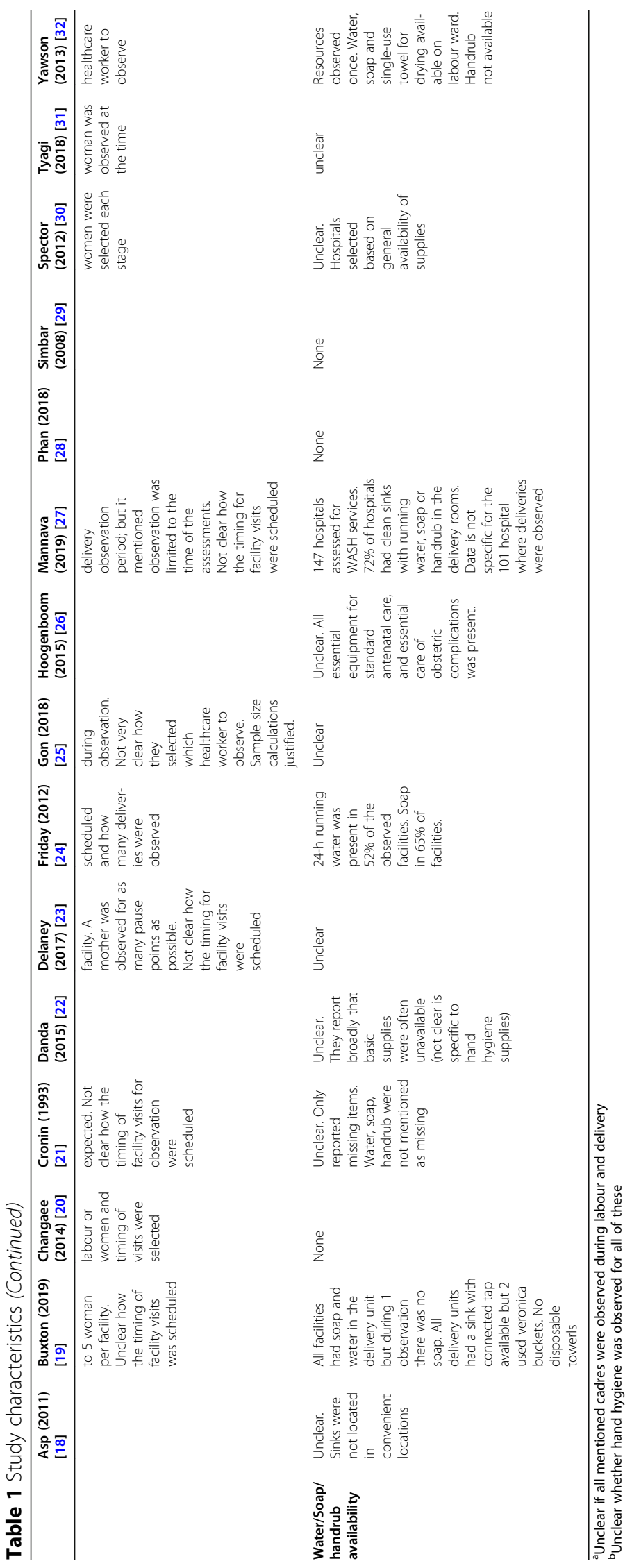


1. Was sampling of the unit of observation described? How a) the unit was sampled b) timing of facility visits were scheduled (e.g. morning vs afternoon)

2. Were any measures to check or enhance the quality of data collection described? 3. a) Were the professional background of observers described? \& b) Did they work in the study facilities?

4. Was the agreement between observers estimated (justifiable only if study clearly used 1 observer)?

5. Was the definition of hand hygiene compliance reported clearly?

6. Was the tool used for data collection a) described and b) available?

7. Did the authors attempt to conceal the study aim from the participants?

8. Were statistical procedure described?

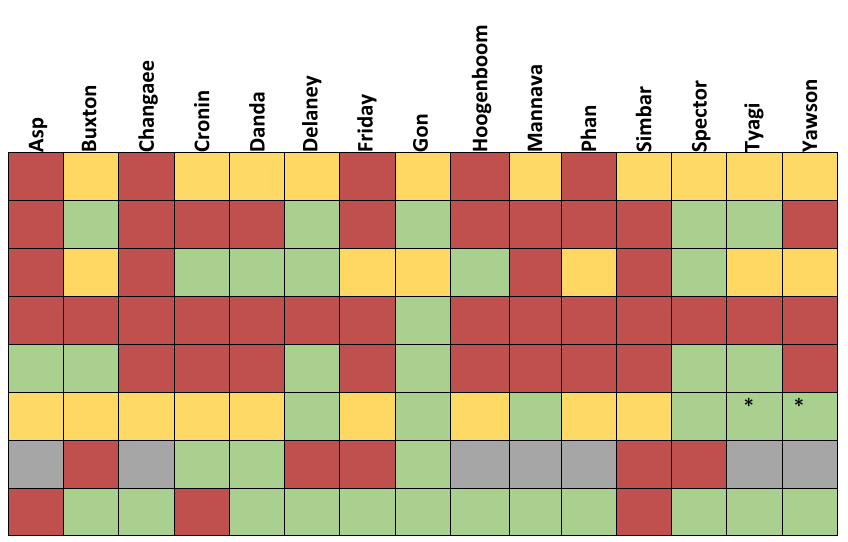

*Modified version of the tool is not available

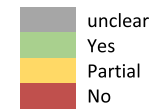

Fig. 2 Risk of bias and quality assessment

Spector et al. [30] attempted to examine agreement between observers - specifically, they reported that periodic assessments were used to confirm that data collectors achieved $100 \%$ concordance on a sample of three observations. Yawson and Hesse only report that different pairs of technical personnel visited the unit each day in order to limit intra-observer bias [32].

\section{Definition of outcome}

Hand hygiene compliance was not defined clearly in most studies. Each definition is reported in detail in Table 2. Some studies did not report whether soap use or handrub was necessary to achieve adequate hand hygiene and did not refer to guidelines that specifically do [20, 22, 24, 26, 27, 29]; in addition often studies did not report if other aspects of hand hygiene such as the sequence of actions preceding or following hand washing/ rubbing, technique or duration were assessed in the summative compliance estimates - except for Gon et al. and Buxton et al. We describe here the studies where definitions presented additional anomalies. Yawson and Hesse, and Phan et al. mentioned that they followed the hand hygiene guidelines by the WHO but it was not clear which aspects of the guidelines they included. Buxton et al. also mentioned that they followed the WHO guidelines but created their own categories of hand hygiene ranging from the least hygienic (category 5) to the most hygienic (category 1 ) which included hand washing with soap, new gloves applied and no potential recontamination. Cronin et al., Danda et al., Friday et al. and Hoogenboom et al. chose a less informative definition of hand hygiene compliance because their denominator referred to whole individuals, group of individuals or facilities rather than specific patient-healthcare worker interactions (e.g. hand washed at least once or at least one birth attendant washed hands). In Changaee et al., it was not clear how they calculated their estimate of desirable hand washing.

Another aspect of the definition is the type of hand hygiene opportunity (when hand hygiene should occur). The WHO hand hygiene guidelines refer to five key hand hygiene opportunities: before clean/clean procedures, after exposure to body fluids, before touching the patient, after touching the patient, after touching the patient's surrounding. Studies did not always report what the type of contact (before vs. after; contact with intact skin i.e. "touching a patient" or non-intact/mucous membrane i.e. clean/aseptic procedures). Indeed, Changaee et al., and Simbar et al. were contacted for further information on their hand hygiene definition as it was unclear if it was before after the procedure/contact, but did not reply [29, 32]. Further enquires were also made to Yawson and Hesse, and Friday et al. on their definitions but with no reply [24, 32]. Another unclear area is what procedures during labour or delivery were captured. Studies that clearly outline this are Gon et al. and Buxton et al. [19, 25]

\section{Hand hygiene compliance estimates during labour and delivery}

We extracted estimates that were clearly for aseptic procedures, and estimates for which this was not clear or where aseptic procedures were not the exclusive focus. Definitions across the studies were extremely heterogeneous and hence we did not combine their estimates; compliance estimates varied from 0 to $100 \%$. Spector et al. reported a baseline compliance of $1.3 \%$ before vaginal examinations during admission and 10.6\% before 


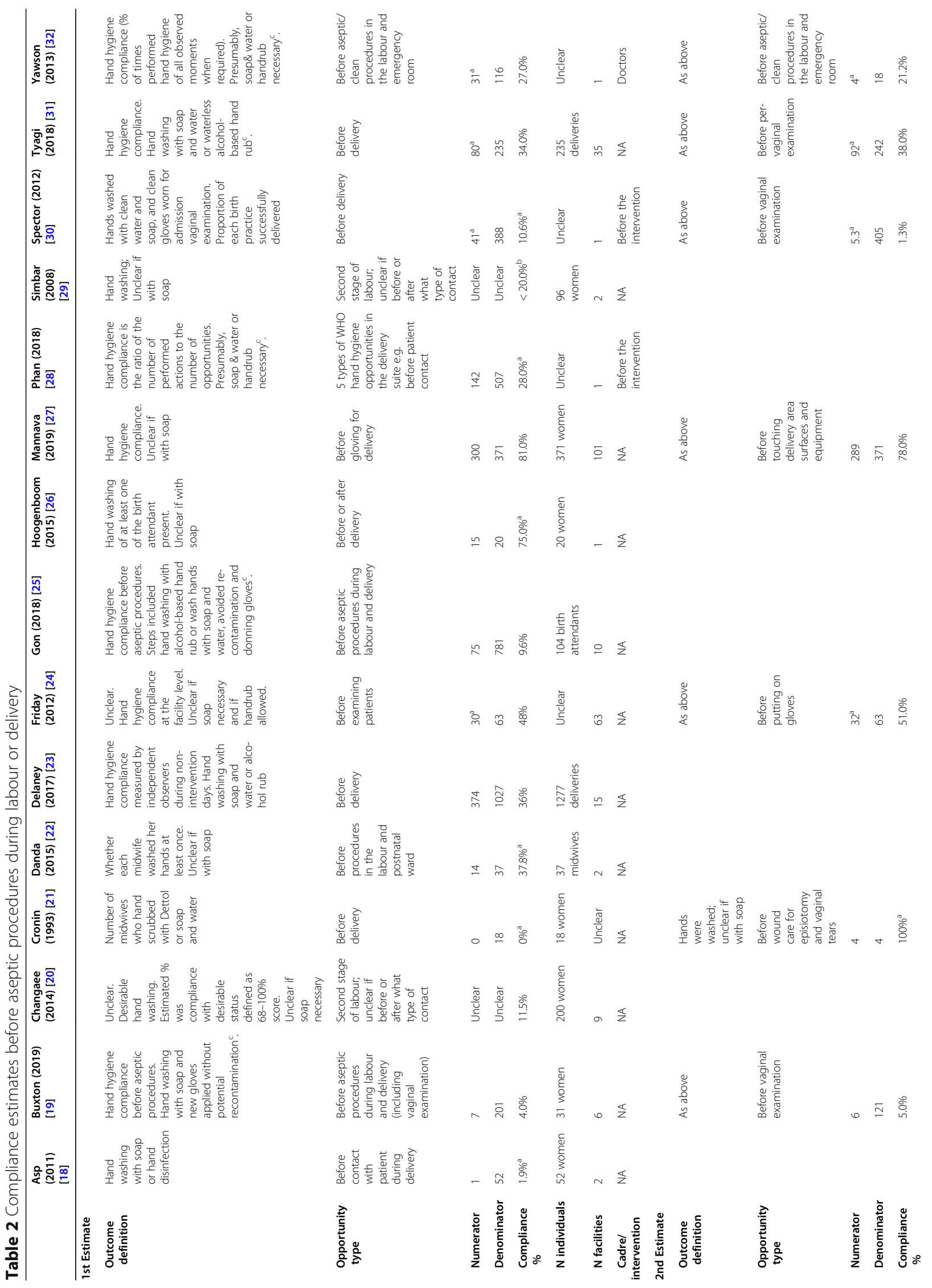




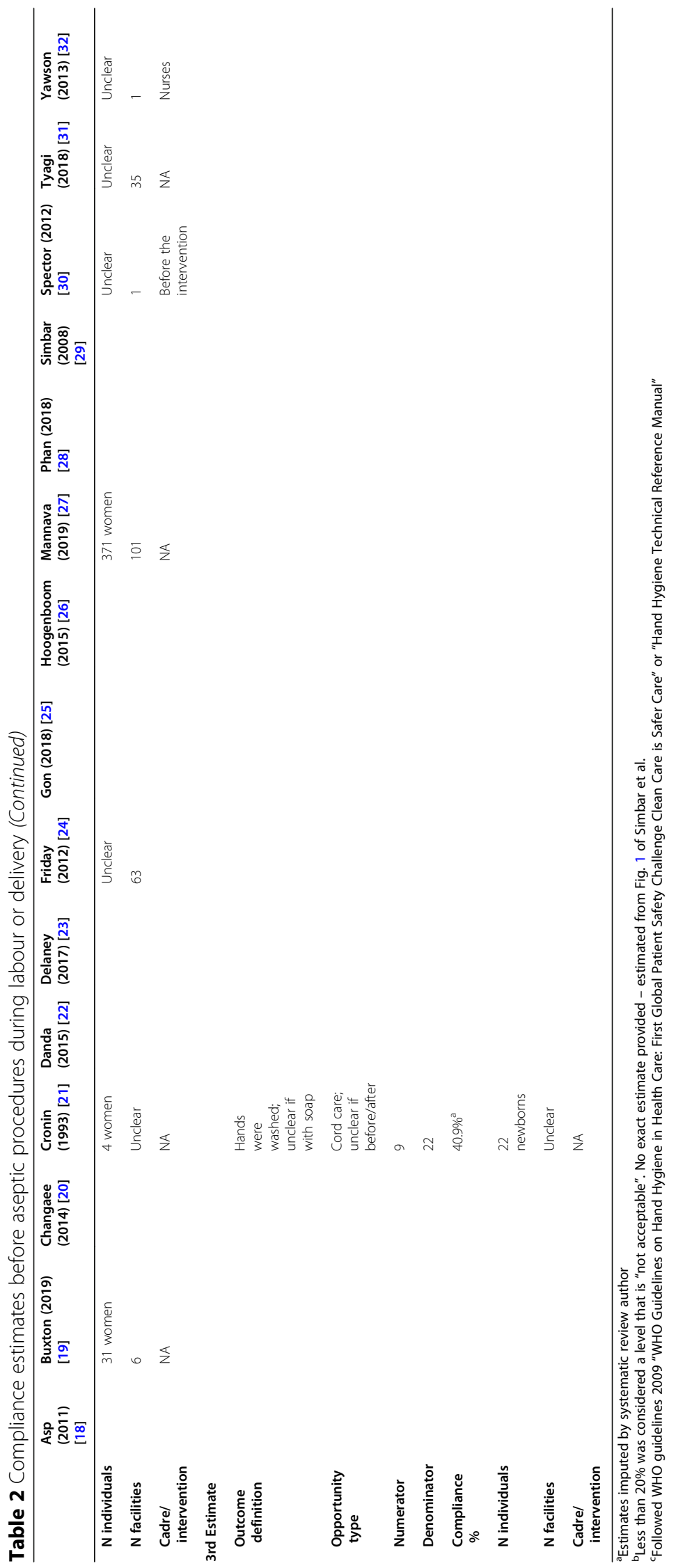


deliveries [30]. A follow up study of the same intervention by Delaney et al. reported compliance before delivery at 36\% after 2 months of intervention measured by independent observers during non-intervention days [23]. Buxton et al. found an overall compliance of $4 \%$ before aseptic procedures during labour and delivery, and a compliance of $5 \%$ before vaginal examination [19]. Gon et al. reported overall compliance with hand rubbing/washing, glove use and avoiding recontamination in $9.6 \%$ of opportunities before aseptic procedures during labour and delivery [25]. Yawson and Hesse reported hand hygiene compliance before aseptic procedures across both the labour and emergency room (we assumed that the emergency room was primarily dedicated to pregnant women); among doctors, compliance was $27.0 \%$, whereas among nurses it was $21.2 \%$ [32]. Phan et al. reported the baseline compliance to be $28 \%$ across five types of WHO hand hygiene opportunities (before patient contact, before aseptic task etc.) observed in the delivery suite [28]. Mannava et al. reported a compliance $81 \%$ before gloving for delivery [27]. Simbar et al. [29] and Changaee et al. [20] reported on compliance during second stage of labour, although it was unclear whether compliance was before or after interaction with the patient or which type of interaction i.e. aseptic procedure, touching the patient. Simbar et al. reported a compliance level below $20.0 \%$, which they describe as unacceptable [29]. We could not interpret the estimate by Chanagaee et al. because the definition of compliance was ambiguous [20]. Asp et al. report a compliance of $1.9 \%$ before contact with patient during delivery or immediate postpartum; it is unclear if this includes aseptic procedures or not [18]. Hoogenboom et al. found that in $75.0 \%$ of deliveries, either before or after the delivery, at least one birth attendant present hand washed [26]. Danda et al. reported compliance before procedures (not clear what type) across the labour and postnatal wards - here, $37.8 \%$ of midwives washed their hands at least once [22]. Friday et al. measured compliance before examining patients in the labour ward (48\%) and before putting on gloves (51\%). However, the compliance represents the percentage of facilities, rather than opportunities or individuals, that comply [24]. Finally, Cronin et al. reported that the midwives scrub hands in none of the 18 deliveries they observed (currently this practice is not necessary before delivery); however, all used either water and soap, or Dettol to perform hand hygiene [21]. All the four observations of wound care in this study were preceded by hand washing $(100 \%)$ but only $40.9 \%$ of the cord-care observations (not clear if before or after cord care).

Table 3 describes the estimates extracted related to "before aseptic procedures" opportunities, from the smallest to the largest, as well as whether we considered their sample size adequate, their definition sufficiently good and whether the authors provided isolated estimates specifically for opportunities before aseptic procedures during labour and delivery. Five studies presented better definitions and larger sample sizes, and were specific to aseptic procedures during labour and birth: Spector et al. [30]; Gon et al. [25]; Buxton et al. [19]; Tyagi et al. [31]; Delaney et al. [23].

\section{Technique and duration of hand hygiene, and avoiding recontamination}

Only three studies [21, 25, 32] reported on aspects of hand hygiene quality such as technique and duration. Cronin et al. reported qualitatively that hand washings were generally not timed (not within the expected duration). Yawson and Hesse reported that on the labour ward, $50 \%$ or more of staff used soap and running water for hand washing, and dried hands with clean single use towels. Less than $50 \%$ washed hands for $40-60 \mathrm{~s}$, or cleaned hands with alcohol handrub, or performed the appropriate handwashing technique [32]. Gon et al. reported the level of adequate rubbing/washing technique at $30.7 \%$ [25] defined as one of the hand gesture required by the WHO technical reference manual [35] i.e. "right palm over left dorsum with interlaced fingers and vice versa"; adequate duration was at $14.6 \%$ defined as $\geq 10 \mathrm{~s}$ based on the local guidelines for infection prevention [25].

Cronin et al. discuss qualitatively the concept of avoiding hand or glove recontamination before a procedure. This is a quote from their article.

"frequent breaks in technique included ... the midwife's gloved hands touching the patient's bed, leg, abdomen, and perineal pad before the delivery." [37].

Gon et al. defined recontamination of hands or gloves as any touch on potentially contaminated surfaces within the workflow after glove donning or hand rubbing/washing when preparing for a an aseptic procedure e.g. touching an unclean delivery surface, unclean hand-drying material, the woman and newborn outside the defined patient zone, the woman's bed, trolley, unclean objects used during hand hygiene, and other unclean surfaces, unless classified as outside the workflow and provide an exhaustive list of these actions and that of patient zone within which touching surfaces is allowed [25]. They report that birth attendants risked recontaminating their hands or gloves in $45.3 \%$ of the opportunities when rubbing/washing or glove donning occurred [25].

Buxton et al. reported avoiding recontamination as part of hand hygiene compliance in the most hygienic 
Table 3 Selected compliance estimates summarised

\begin{tabular}{|c|c|c|c|c|c|}
\hline $\begin{array}{l}\% \\
\text { Compliance }\end{array}$ & Author & Type of opportunity & $\begin{array}{l}\text { Sample } \\
\text { size }\end{array}$ & Definition & $\begin{array}{l}\text { Specific estimate before aseptic proc. during labour } \\
\text { and delivery }\end{array}$ \\
\hline 0 & Cronin [21] & Before delivery & Small & Suboptimal & No \\
\hline 1.3 & Spector [30] & Before vaginal exam. & Adequate & Good & Yes \\
\hline 1.9 & Asp [18] & Before contact & Adequate & Suboptimal & No \\
\hline 4.0 & Buxton [19] & Before aseptic procedures & Adequate & Good & Yes \\
\hline 5.0 & Buxton [19] & Before vaginal examination & Adequate & Good & Yes \\
\hline 9.6 & Gon [25] & Before aseptic procedures & Adequate & Good & Yes \\
\hline 10.6 & Spector [30] & Before delivery & Adequate & Good & Yes \\
\hline 11.5 & Changaee [20] & II stage of labour & Adequate & Suboptimal & No \\
\hline$<20$ & Simbar [29] & II stage of labour & Adequate & Suboptimal & No \\
\hline 21.2 & Yawson [32] & Before aseptic (doct.) & Adequate & Satisfactory & Unclear $^{\mathrm{a}}$ \\
\hline 27.0 & Yawson [32] & Before aseptic (nurs.) & Adequate & Satisfactory & Unclear $^{a}$ \\
\hline 28.0 & Phan [28] & All 5 types of opp. & Adequate & Satisfactory & No \\
\hline 34.0 & Tyagi [31] & Before delivery & Adequate & Good & Yes \\
\hline 36.0 & Delaney [23] & $\begin{array}{l}\text { Before delivery (independent } \\
\text { observers) }\end{array}$ & Adequate & Good & Yes \\
\hline 37.8 & Danda [22] & Before procedures & Small & Suboptimal & No \\
\hline 38.0 & Tyagi [31] & Before vaginal examination & Adequate & Good & Yes \\
\hline 40.9 & Cronin [21] & During cord care & Small & Suboptimal & Yes \\
\hline 48.0 & Friday [24] & Before examining patients & Unclear & Suboptimal & Unclear \\
\hline 51.0 & Friday [24] & Before putting on gloves & Unclear & Suboptimal & Unclear \\
\hline 75.0 & $\begin{array}{l}\text { Hoogenboom } \\
{[26]}\end{array}$ & During delivery & Small & Suboptimal & No \\
\hline 78.0 & Mannava [27] & $\begin{array}{l}\text { Before touching any delivery areas } \\
\text { or surface }\end{array}$ & Adequate & Satisfactory & Yes \\
\hline 81.0 & Mannava [27] & Before gloving for delivery & Adequate & Satisfactory & Yes \\
\hline 100 & Cronin [21] & Before wound care & Small & Satisfactory & Yes \\
\hline
\end{tabular}

${ }^{a}$ Emergency room may not only cater for labouring women

category but did not specify the definition of what behaviours are included in recontamination [19].

\section{Interventions, effect size for hand hygiene determinants and ICC}

Three studies report interventions aimed at increasing hand hygiene compliance. Two studies relied on a prepost intervention design, without randomization or control wards; one only reported the intervention period without baseline. The three studies reported on interventions including several components - two of these studies discuss the same intervention. Phan et al. [28] tested an educational program on hand hygiene provided to healthcare workers over two $3 \mathrm{~h}$ sessions. The educational model used experiential learning and incorporated novel techniques of learning that allowed for consideration of past hand hygiene experiences. Fifty two out of 53 healthcare staff in the delivery suite participated in the intervention. The intervention improved hand hygiene overall in the selected wards, but the effect was largest in the delivery suite increasing from 28 to $61.8 \%$ across all five types of WHO hand hygiene opportunities [28]. The improvement was sustained over a period of 6 months of post intervention follow-up. Given the nature of the intervention, we assumed that participants were not blinded to the aim of the intervention.

Spector et al. tested a four-components childbirth safety program based on the WHO Safe Childbirth Checklist [30]. After the intervention, hand hygiene compliance increased respectively from 1.3 to $97.8 \%$ before vaginal examination during admission and from 10.6 to $99.5 \%$ before delivery. The checklist included prompts on elements of hand hygiene; therefore, the healthcare workers were not blinded to the aim of the intervention. Delaney et al. [23] also describes the introduction of the WHO's Safe Childbirth Checklist. This was part of a large randomised control trial, but the article included here focuses on the 60 facilities that 
received the intervention. There is no control or baseline group for comparing hand hygiene without the intervention. The main comparison is between the first month of intervention and the latter 7-8 months carried out by the same peer-coaches who run the intervention - compliance before delivery was respectively $76 \%$ and $94 \%$. The independent assessment of hand hygiene described above showed compliance at $36 \%$ between 2 and 5 months of the intervention period. Given the presence of peer coaching, participants were not blinded to the aim of the intervention.

A few studies looked quantitatively at the association between potential determinants and hand hygiene compliance (measured via observation or other objective method) - but none of these were individual level determinants except for cadre. These appear to be all unadjusted associations. Mannava et al. reported that hand hygiene compliance before touching any delivery surfaces was lower in tertiary hospitals at $71 \%$, vs $83 \%$ for first-level referral hospitals ( $p$-value $<0.001)$, and higher in hospitals where all delivery rooms had soap and a sink with water compared to hospitals where needed supplies was not available in all rooms ( $50 \%$ vs $39 \%, p$-value $=$ 0.29) [27]. Buxton et al. tested the association between hand hygiene compliance and cadre, national state, and facility type - these were not found to be associated; they do find an association with shift - with the morning shift having higher compliance compared to the afternoon $(p$-value $=0.0034)$ and night $(p$-value $=0.008)$ [19] Tyagi et al. described hand hygiene compliance by facility type, reporting a compliance of $100 \%$ in private facilities compared to $27 \%$ in public facilities ( $p$-value $=$ 0.011) [31]. They do not find an association with facility level and facility load [31]. Gon et al. report that hand hygiene compliance did not vary much by observer or by shift, indeed the confidence intervals overlapped across the of these categories [25].

With regards to the ICC, we present here the results we gathered from studies with the larger sample size and clearer definitions, involving more than two facilities, and where authors replied to our request. Estimates of rho in Buxton et al. [19] and Gon et al. [25], are both closer to 0 than 1 indicating that variance within facilities appear higher than between facilities (Table 4).
ICC for the variation between and within individuals is also provided by Gon et al. and reports higher variance within than between individuals [25].

\section{Discussion}

We performed a systematic review of published studies reporting estimates of birth attendants' hand hygiene compliance conducted in healthcare facilities in LMICs. We found fifteen studies that met our inclusion criteria. Hand hygiene compliance estimates were extremely diverse, ranging from 0 to $100 \%$; the heterogeneity in definitions of hand hygiene did not allow us to combine or compare these meaningfully. Four studies (Cronin et al., Hoogenboom et al., Friday et al., and Mannava et al.) reported higher compliance. Except for Mannava et al., these with higher compliance also had a very small or unclear sample, and used an individual level or group level definition for the denominator rather than the number of patient-attendant interactions (hand hygiene opportunities) as recommended by the WHO hand hygiene guidelines $[21,22,26]$. The studies $[19,23,25,28$, 30-32] with larger sample sizes and clearer definitions suggest compliance to hand hygiene before aseptic procedures to be low, between 1.3 and $38.0 \%$. We have three estimates for hand hygiene before vaginal examination which spans between 1.3\% [30] and 38\% [31]; and we have five estimates for hand hygiene before labour/ delivery-related procedures spans between 4 and 36\% [23]. Overall, the quality of the included studies was particularly compromised by poorly described sampling methods and definitions.

The studies included were published in the last 18 years and spanned 14 countries between Sub-Saharan Africa, South East Asia and the Middle East. Four studies only included one facility, limiting their generalizability. The supplies of key hand hygiene infrastructure were poorly described, except in four studies. The quality of the studies included was generally poor with a high risk of bias with a few exceptions. The weakest aspect of the studies was their description of the sampling strategy, as most studies did not describe how the unit of observation was sampled (whether women, healthcare workers or specific procedures). Also, the reported definitions of hand hygiene were often

Table 4 ICC results

\begin{tabular}{lll}
\hline & Buxton et al. [19] & Gon et al. [25] \\
\hline Outcome is hand hygiene during: & Before aseptic procedures during labour/delivery & Before aseptic procedures during labour/delivery \\
Facilities & 6 & 10 \\
Numerator & 7 & 75 \\
Denominator & 201 & 781 \\
Rho & $<0.0001$ & 0.13 \\
\hline
\end{tabular}


incomplete. For most studies it was unclear whether the use of soap was a necessary condition to achieve hand washing compliance. In addition, the type of hand hygiene opportunity was often poorly described i.e. before or after the interaction with the patient; aseptic procedures vs. contact with the patient intact skin. Finally, in four studies the denominator did not rely on patientworker interactions but on the overall performance of an individual or a group, or on the number facilities were hand hygiene was observed. This finding, of poor methods in conducting and reporting of observational studies on hand hygiene and more broadly of healthcare workers, was reported elsewhere [6, 36].

Beyond the basic aspects of quality required for any observational study and described by the STROBE guidelines [15], future studies focusing on hand hygiene during labour and delivery should design and report the following more clearly:

a) what sampling strategy was used to observe either workers, women, or patient-worker interactions; and how facilities visits were scheduled;

b) the methods used to ensure the quality of data collection in the study e.g. data monitoring

c) the inter-observer agreement where multiple observers are employed;

d) the definition of hand hygiene using the WHO hand hygiene guidelines [37] (i.e. soap necessary for hand washing; which type of hand hygiene opportunity e.g. before vs. after, touching intact skin vs. aseptic procedure; denominator based on patient-worker interactions rather than individual or group level performance; types of procedures involved in the aseptic procedure; sequence of actions required to comply to hand hygiene);

Our findings of low birth attendants' hand hygiene compliance are consistent with other systematic reviews or multi-country studies in LMICs of hand hygiene among healthcare workers more generally, which report compliance estimates ranging from 22 to $35 \%$ during nonintervention periods [38, 39]. Similarly to these studies, our estimates point to a slight lower compliance in LMICs compared to high-income settings. With approximately 140 million women delivering worldwide, most of which are in LMICs and at least half of which occur in healthcare facilities where quality of care is suboptimal, these low estimates of hand hygiene compliance during labour/delivery are worrisome $[5,40,41]$. If correct, these estimates pose a substantial risk to infection prevention during birth in LMICs where both mothers and newborns are still largely affected by infection $[1,2,42]$.

None of the included studies specifically investigated the wide range of individual determinants of hand hygiene compliance - except for cadre examined in one study. Four however report compliance estimates by study or facility characteristics. Three studies [30, 32] investigated the effect of two different interventions on hand hygiene, a checklist on quality of care at birth and an education program. Both were successful in increasing substantially the hand hygiene compliance during labour/delivery. Given the nature of their study design pre-post intervention without a control ward, or without baseline, and with study participants who are no blinded - these interventions tell us more about the feasibility of these interventions in these specific contexts compared to anything conclusive about their scope for improving hand hygiene more widely in LMICs. With regards to ICC, from 2 studies we find that variation is greater within than between facilities.

Our systematic review covered four separate databases, has a clearly reported search strategy adapted from previous systematic reviews on the topic, did not pose any restrictions based on language, and used independent double full text screening and article extraction. A potential weakness is that our search might have missed articles which included hand hygiene in the broader framework of quality of care during birth or infection prevention and control and which did not mention hand hygiene in their title or abstract. We did not assess publication bias, but this would be more of an issue for intervention studies that found negative results for example than for observational studies reporting on compliance estimates. Finally, the set of health care facilities included in this systematic review is unlikely to represent health care facilities across LMICs. Without random sampling from the reference population of health care facilities, estimates of hand hygiene may be subject to selection bias stemming from researchers non-random decisions about which facilities to study. For example, researchers may be more likely to sample from higher volume facilities where deliveries are frequent than to sample from lower volume facilities. Studies suggest that higher volume facilities are better equipped for attending deliveries, but they maybe more prone to crowding which in turn makes hand hygiene more challenging [43]. Only Gon et al., Mannava et al., Tyagi et al. [25, 27, 31] can be regarded representative of the reference population which they targeted, respectively: high-volume labour wards in Zanzibar, hospitals implementing EENC in the countries included from South East Asia, hospitals with a newborn unit in Andhra Pradesh and Telengana regions of India who did not receive a quality improvement intervention. It is hard to make this inference for Friday et al. because of their group level definition of hand hygiene. [24] 


\section{Conclusions}

In conclusion, we found fifteen articles reporting the hand hygiene compliance of healthcare workers during labour and delivery in LMICs. Compliance including before aseptic procedures opportunities for studies with larger sample sizes and clear definitions was low, ranging between 1 and $38 \%$. This is an opportunity for infection prevention reduction during birth in LMICs facilities since effective interventions in this area are likely to reduce infection rate among mothers and newborns. We also found that the quality of many studies was suboptimal. In particular, future studies of hand hygiene compliance during the labour ward should be designed with better sampling frame, assess inter-observer agreement, use measures to improve quality of data collection and report their hand hygiene definitions clearly.

\section{Supplementary Information}

The online version contains supplementary material available at https://doi. org/10.1186/s12913-020-05925-9.

Additional file 1. "Systematic review search strategy" - it includes the search strategy for each database used in our review.

Additional file 2. "PRISMA 2009 Checklist" - It includes the details of our manuscript against the PRISMA checklist.

\section{Abbreviations}

CONSORT: Consolidated Standards of Reporting Trials; HICs: High Income Countries; ICC: Intra-cluster correlation LMICs; PRISMA: Preferred Reporting Items for Systematic Reviews and Meta-Analyses; STROBE: Strengthening the Reporting of Observational studies in Epidemiology; WHO: World Health Organisation

\section{Acknowledgements}

We thank Kerry Wong, Sophie Sarassat, and Andreia Leite for their help in translating articles from Chinese, French and Portuguese respectively. We also thank the librarian, Jane Falconer, at the London School of Hygiene and Tropical Medicine for her suggestions on the search strategy. Finally, we thank Sandra Virgo for running the updated search strategy in April 2018.

\section{Authors' contributions}

Conceived and designed the study: GG OC. Analysed the data: GG first reviewer, MDB and LD second reviewers. Wrote the paper: GG MDB SN OC LD. Data interpretation: GG MDB SN OC LD. The authors have read and approved the manuscript.

\section{Funding}

The Soapbox Collaborative contributed by funding the corresponding author, GG.

The writing of this paper provided part of the background. needed for the HANDS study funded by the Medical Research Council -PHIND scheme, award number MR/N015975/1, and the CLEAN Study, funded by the United Kingdom Joint Global Health Trials (Wellcome, MRC, DFID, and DOH), award number MR/R019274/1. SN is supported by an award jointly funded by the UK Medical Research Council (MRC) and the UK Department for International Development (DFID) under the MRC/DFID Concordat agreement which is also part of the EDCTP2 programme supported by the European Union. Grant Reference MR/K012126/1. None of the funding bodies had any role in the design of the study or the collection, analysis, and interpretation of the data, or in writing the manuscript.

All authors have read and approved the manuscript.

\section{Availability of data and materials}

All data generated or analysed during this study are included in this published article.

\section{Ethics approval and consent to participate}

Not applicable.

\section{Consent for publication}

Not applicable.

\section{Competing interests}

The authors declare that they have no competing interests.

\section{Author details}

${ }^{1}$ London School of Hygiene and Tropical Medicine, Keppel Street, London WC1E 7HT, UK. ${ }^{2}$ Brunel University London, Department of Life Sciences, Uxbridge, UK. ${ }^{3}$ Independent researcher, Turin, Italy.

Received: 23 December 2019 Accepted: 15 November 2020

Published online: 03 December 2020

\section{References}

1. Kassebaum NJ, Bertozzi-Villa A, Coggeshall MS, Shackelford KA, Steiner C, Heuton KR, et al. Global, regional, and national levels and causes of maternal mortality during 1990-2013: a systematic analysis for the global burden of disease study 2013. Lancet. 2014;384:980-1004.

2. Oza S, Lawn JE, Hogan DR, Mathers C, Cousens SN. Neonatal cause-of-death estimates for the early and late neonatal periods for 194 countries: 20002013. Bull World Health Organ. 2015;93:19-28.

3. Gould IM. Alexander Gordon, puerperal sepsis, and modern theories of infection control--Semmelweis in perspective. Lancet Infect Dis. 2010;10: 275-8.

4. Semmelweis I. The etiology, concept and prophylaxis of childbed fever. Madison: The University of Wisconsin Press; 1983.

5. Campbell OMR, Calvert C, Testa A, Strehlow M, Benova L, Keyes E, et al. The scale, scope, coverage, and capability of childbirth care. Lancet. 2016;388: 2193-208.

6. Erasmus V, Daha TJ, Brug H, Richardus JH, Behrendt MD, Vos MC, et al. Systematic review of studies on compliance with hand hygiene guidelines in hospital care. Infect Control Hosp Epidemiol. 2010;31:283-94.

7. Vindigni SM, Riley PL, Jhung M. Systematic review: handwashing behaviour in low- to middle-income countries: outcome measures and behaviour maintenance. Tropical Med Int Health. 2011;16:466-77.

8. Luangasanatip N, Hongsuwan M, Limmathurotsakul D, Lubell Y, Lee AS, Harbarth $S$, et al. Comparative efficacy of interventions to promote hand hygiene in hospital: systematic review and network meta-analysis. The BMJ. 2015;351:h3728.

9. Gould DJ, Moralejo D, Drey N, Chudleigh JH, Taljaard M. Interventions to improve hand hygiene compliance in patient care. Cochrane Database Syst Rev. 2017;9:CD005186.

10. Schweizer ML, Reisinger HS, Ohl M, Formanek MB, Blevins A, Ward MA, et al. Searching for an optimal hand hygiene bundle: a meta-analysis. Clin Infect Dis. 2014;58:248-59.

11. Cross S, Afsana K, Banu M, Mavalankar D, Morrison E, Rahman A, et al. Hygiene on maternity units: lessons from a needs assessment in Bangladesh and India. Glob Health Action. 2016;9. https://doi.org/10.3402/gha.v9.32541.

12. Gon G, Restrepo-Méndez MC, Campbell OMR, Barros AJD, Woodd S, Benova $L$, et al. Who delivers without water? A multi country analysis of water and sanitation in the childbirth environment. PLoS One. 2016;11:e0160572.

13. Huis A, van Achterberg T, de Bruin M, Grol R, Schoonhoven L, Hulscher M. A systematic review of hand hygiene improvement strategies: a behavioural approach. Implement Sci. 2012;7:92.

14. Gould DJ, Moralejo D, Drey N, Chudleigh JH, Taljaard M. Interventions to improve hand hygiene compliance in patient care. Cochrane Database Syst Rev. 2017;9:CD005186. https://doi.org/10.1002/14651858.CD005186.pub4.

15. STROBE Initiative Group. STROBE checklist for cohort, case-control, and cross-sectional studies (combined); 2007. https://www.strobe-statement.org/ index.php?id=strobe-group0. Accessed 14 Dec 2017.

16. Sanderson S, Tatt ID, Higgins JPT. Tools for assessing quality and susceptibility to bias in observational studies in epidemiology: a systematic review and annotated bibliography. Int J Epidemiol. 2007;36:666-76. 
17. PRISMA. http://www.prisma-statement.org/. Accessed 24 Apr 2017.

18. Asp G, Sandberg J, Ezechi O, Pettersson KO. Challenges of immediate newborn care in maternity units in Lagos, Nigeria: an observational study. J Obstet Gynaecol. 2011;31:612-6.

19. Buxton H, Flynn E, Oluyinka O, Cumming O, Esteves Mills J, Shiras T, et al. Hygiene during childbirth: an observational study to understand infection risk in healthcare facilities in Kogi and Ebonyi states, Nigeria. Int J Environ Res Public Health. 2019;16:1301

20. Changaee F, Simbar M, Irajpour A, Akbari S. Quality assessment of Peripartum care. Iran Red Crescent Med J. 2014;16. https://doi.org/10.5812/ ircmj.9069.

21. Cronin WA, Quansah MG, Larson E. Obstetric infection control in a developing country. J Obstet Gynecol Neonatal Nurs. 1993;22:137-44.

22. Danda G, Dube K, Dube P, Mudokwenyu-Rawdon C, Bedwell C. An observational study of midwives' practices to prevent peripartum sepsis in Zimbabwe. Afr J Midwifery Womens Health. 2015;9:17-21.

23. Marx Delaney M, Maji P, Kalita T, Kara N, Rana D, Kumar K, et al. Improving adherence to essential birth practices using the $\mathrm{WHO}$ safe childbirth checklist with peer coaching: experience from 60 public health facilities in Uttar Pradesh, India. Glob Health Sci Pract. 2017;5:217-31.

24. Friday O, Edoja O, Osasu A, Chinenye N, Cyril M, Lovney K, et al. Assessment of infection control practices in maternity units in southern Nigeria. Int J Qual Health Care. 2012;24:634-40.

25. Gon G, de Bruin M, de Barra M, Ali SM, Campbell OM, Graham WJ, et al. Hands washing, glove use, and avoiding recontamination before aseptic procedures at birth: a multicenter time-and-motion study conducted in Zanzibar. Am J Infect Control. 2019;47:149-56.

26. Hoogenboom G, Thwin MM, Velink K, Baaijens M, Charrunwatthana P, Nosten F, et al. Quality of intrapartum care by skilled birth attendants in a refugee clinic on the Thai-Myanmar border: a survey using WHO safe motherhood needs assessment. BMC Pregnancy and Childbirth. 2015;15:17.

27. Mannava P, Murray JC, Kim R, Sobel HL. Status of water, sanitation and hygiene services for childbirth and newborn care in seven countries in East Asia and the Pacific. J Glob Health. 9. https://doi.org/10.7189/jogh.09. 020430.

28. Phan HT, Tran HTT, Tran HTM, Dinh APP, Ngo HT, Theorell-Haglow J, et al. An educational intervention to improve hand hygiene compliance in Vietnam. BMC Infect Dis. 2018;18. https://doi.org/10.1186/s12879-018-3029-5.

29. Simbar M, Ghafari F, Zahrani ST, Majd HA. Assessment of quality of midwifery care in labour and delivery wards of selected Kordestan medical Science University hospitals. Int J Health Care Qual Assur. 2009;22:266-77.

30. Spector JM, Agrawal P, Kodkany B, Lipsitz S, Lashoher A, Dziekan G, et al. Improving quality of Care for Maternal and Newborn Health: prospective pilot study of the WHO safe childbirth checklist program. PLoS One. 2012;7: e35151.

31. Tyagi M, Hanson C, Schellenberg J, Chamarty S, Singh S. Hand hygiene in hospitals: an observational study in hospitals from two southern states of India. BMC Public Health. 2018;18. https://doi.org/10.1186/s12889-018-62196.

32. Yawson AE, Hesse AAJ. Hand hygiene practices and resources in a teaching hospital in Ghana. J Infect Dev Ctries. 2013;7:338-47.

33. Christensson K, Pettersson KO, Bugalho A, null C, Manuela M, Dgedge C, et al. The challenge of improving perinatal care in settings with limited resources. Observations of midwifery practices in Mozambique. Afr J Reprod Health. 2006;10:47-61.

34. MCHIP. Quality of antenatal and delivery care services in six countries in sub-Saharan Africa. Washington, DC: MCHIP; 2013.

35. WHO. Hand hygiene technical reference manual: WHO; 2009. http://apps. who.int/iris/bitstream/handle/10665/44196/9789241598606_eng.pdf; jsessionid=C23CC7AC25035E11B1F80D3012E835E4? sequence $=1$. Accessed 27 Nov 2017.

36. Jeanes A, Coen PG, Gould DJ, Drey NS. Validity of hand hygiene compliance measurement by observation: a systematic review. Am J Infect Control 2018;0. doi:https://doi.org/10.1016/j.ajic.2018.08.004.

37. World Health Organization. WHO guidelines on hand hygiene in health care: first global patient safety challenge clean care is safer care. Geneva: WHO; 2009. http://www.who.int/gpsc/5may/tools/9789241597906/en/. Accessed 26 Nov 2015

38. Allegranzi B, Gayet-Ageron A, Damani N, Bengaly L, McLaws M-L, Moro M-L, et al. Global implementation of WHO's multimodal strategy for improvement of hand hygiene: a quasi-experimental study. Lancet Infect Dis. 2013;13:843-51.

39. Amazian K, Abdelmoumène T, Sekkat $S$, Terzaki S, Njah M, Dhidah L, et al. Multicentre study on hand hygiene facilities and practice in the Mediterranean area: results from the NosoMed network. J Hosp Infect. 2006; 62:311-8.

40. Graham W, Woodd S, Byass P, Filippi V, Gon G, Virgo S, et al. Diversity and divergence: the dynamic burden of poor maternal health. Lancet. https:// doi.org/10.1016/S0140-6736(16)31533-1.

41. Montagu D, Yamey G, Visconti A, Harding A, Yoong J. Where do poor women in developing countries give birth? A multi-country analysis of demographic and health survey data. PLoS One. 2011;6:e17155.

42. Seale AC, Mwaniki M, Newton CRJC, Berkley JA. Maternal and early onset neonatal bacterial sepsis: burden and strategies for prevention in subSaharan Africa. Lancet Infect Dis. 2009:9:428-38.

43. Kruk ME, Leslie HH, Verguet S, Mbaruku GM, Adanu RMK, Langer A. Quality of basic maternal care functions in health facilities of five African countries: an analysis of national health system surveys. Lancet Glob Health. 2016;4: e845-55.

\section{Publisher's Note}

Springer Nature remains neutral with regard to jurisdictional claims in published maps and institutional affiliations.
Ready to submit your research? Choose BMC and benefit from:

- fast, convenient online submission

- thorough peer review by experienced researchers in your field

- rapid publication on acceptance

- support for research data, including large and complex data types

- gold Open Access which fosters wider collaboration and increased citations

- maximum visibility for your research: over $100 \mathrm{M}$ website views per year

At BMC, research is always in progress.

Learn more biomedcentral.com/submissions 